\title{
Radiation-induced Demyelination and Remyelination in the Central Nervous System: A Literature Review
}

\author{
LAWRENCE HELSON
}

Sabinsa Pharmaceutical Company, East Winsdor, NJ, U.S.A.

\begin{abstract}
Therapeutic radiation applied to the central nervous system concomitant with or followed by surgery and chemotherapy induces significant pathologic demyelination depending upon tumor volume, dosage, field of treatment, and age of patient, with consequent exacerbation of significant impairment of mental function including personality change, memory deficiencies, confusion, learning difficulties and dementia. These adverse clinical events may be ameliorated by the application of remyelinating measures including nutrition, supplements and pharmaceuticals prophylactically, concomitant with radiation or post-radiation treatment.
\end{abstract}

In 1929, Druckmann published the first description of acute radiation myelopathy (1). He reported that the central nervous system disordered signs and symptoms followed by radiation within weeks but complete recovery was commonly observed. This condition was associated with demyelination (2). A delayed chronic form that developed months after radiation was considered to be secondary to nerve degeneration and vascular insufficiency (3). These were early observations of the sensitivity of the central nervous system to ionizing radiation, which has continued to be used for the prevention of small cell lung cancer metastases to the brain, and as a therapeutic measure for primary or metastatic tumors of the brain. Following surgical diagnostic biopsy or debulking of cerebral tumors (gliomas), current treatment generally consists of focal or whole brain radiation, chemotherapy, and recently, experimental immune therapy $(4,5)$.

Gliomas are uncommon in the general population with similar incidence in males and females (6). However, when they

This article is freely accessible online.

Correspondence to: Lawrence Helson, MD, Sabinsa Pharmaceutical Company, 20 Lake Drive, East Winsdor, NJ 08520, U.S.A. Tel: +1 2155389996, e-mail: 1helson@comcast.net

Key Words: Demyelination, remyelination, radiation review. occur as glioblastomas, they may be extremely aggressive. The median survival of patients with grade IV glioblastoma multiforme is $12-15$ months, and 24-36 months for patients with grade III glioma. The high mortality rate, and limited survival time conferred by these tumors limits therapeutic objectives towards extending survival and improving or maintaining quality of life. Following imaging diagnosis in patients with glioblastoma, surgical biopsy or debulking of tumors is used as initial treatment. However, while this procedure may temporarily diminish symptoms, there is scant evidence that it prolongs survival, and it may also promote an unwanted inflammatory response (7). Ionizing radiation, a second modality of treatment, whether focal or applied to the whole brain extends survival time compared to debulking surgery applied alone. The addition of chemotherapy does not confer a significant extension of overall, or progression-free survival.

With the exception of magnetic resonance imaging, putative disease modifying surgery, chemotherapy and particularly radiation therapy have harmful side effects which can add to or exacerbate tumor-related pre-existing regional and or global cerebral pathologic changes. This can lead to additional acute and/or chronic neurological symptoms. Pre-existing tumor-induced pathological damage in surrounding neural tissue may result from space occupancy and tissue compression depending upon the size and location of the tumor (8), disturbances of the regional vascular supply causing hypoxic-ischemia, and partial to complete demyelination. Secondary to administration of antitumor chemotherapies i.e., methotrexate, thiotepa, $\mathrm{BCNU}$, cisplatin or cytosine arabinoside, following or in combination with ionizing radiation there may occur enhanced oligodendrocyte susceptibility apoptosis and consequent demyelination (9). This is critically important since oligodendrocytes in the central nervous system are responsible for producing the myelin sheath around axons, and if damaged, impact on axonal function $(10,11)$. Experimental data supporting the demyelinating effects of ionizing radiation has been demonstrated in in vitro studies of the apoptotic effects of radiation on cultures of O-2A 
progenitor, immature, and mature oligodendrocytes. In untreated terminally differentiated oligodendrocytes where $15 \%$ of the cells are apoptotic, within one to six hours following 10 Gray radiation, there is an increase in apoptotic cells to $30 \%$, By contrast, irradiation of cultures of $\mathrm{O}-2 \mathrm{~A}$ progenitor or immature oligodendrocytes do not undergo apoptosis (12). Therapeutic radiation of the brain may induce acute, or chronic clinical neurological disorders in over $50 \%$ of patients within two months of treatment with clinical symptoms ranging from asymptomatic, where post-radiation imaging procedures may demonstrate pathologic changes, to significant impairment of mental function including personality change, memory deficiencies, confusion, learning difficulties and dementia. The extent of adverse cerebral effects of radiation-induced demyelination depends upon, the radiation dose, fractionation methods, and whether focal or whole brain fields are administered.

Symptomatic neurological disorders accompanied by brain atrophy resulting from demyelinating processes constitute serious post-radiotherapeutic adverse effects with the greatest risk associated with application of whole brain irradiation in persons greater than 70 years of age (11).

Post-irradiation pathological events include demyelination, proliferation of glial and mononuclear cells, and progression to hyperplasia of the capillary endothelium resulting in reduced cerebral blood flow, perivascular inflammation, diffuse vasogenic edema of the cerebral white matter, necrotic foci, vacuolation, and petechial hemorrhage. These late effects of radiation-induced pathology can be detected by magnetic resonance imaging between six to eight months following initial therapy and continue to evolve in patients who survive for longer periods of time. The characteristic pattern of radiation injury on magnetic resonance imaging is a symmetric, high-signal foci on T2-weighted images in the periventricular white matter with initial sparing of the corpus callosum and the subcortical arcuate fibers. Eventually, a confluent lesion pattern develops. As the pathological process extends to the peripheral arcuate fibers of the white matter, the margins become scalloped. Ultimately atrophy can be demonstrated by enlargement of the ventricles and cortical sulci. In some patients, advancement to the posterior fossa, basal ganglia, and internal capsules, may also occur. These changes are more prevalent with ischemia.

In a case report, six autopsy studies of brain irradiated patients were reviewed neurologically, radiographically, and histo-pathologically. All patients presented progressive disturbances of mental status and consciousness, akinesia, and tremor-like involuntary movement. Histological examination demonstrated swelling and loss of the myelin sheath in the white matter in all patients, and reactive astrocytosis in three of the six patients. These observations suggest that demyelination results from the direct neurotoxic effect of ionizing radiation (12). In contrast to conventional therapeutic radiation, focal high-dose therapy may lead to, radiation necrosis and profound edema, mass effect, and contrast enhancement with gadolinium.

\section{Discussion}

Demyelination following radiation for cerebral tumors poses several problematic issues: 1) The tumor bulk induces a demyelination penumbra in surrounding tissues due to compression, and vascular perturbance; 2) Administering whole brain or focal ionizing radiation adds additional demyelination to the tumor and surrounding tissues; 3 ) Antitumor chemotherapy may further exacerbate demyelination. In the presence of genetic, prenatal or postnatal demyelinating disorders affecting the brain, remyelinating strategies are important to minimize progressive pathological injury due to enhanced demyelination. In planning clinical trials, incorporation of remyelinating strategies can be applied in a preventative care context, or concomitant with demyelinating radiation or drugs or after detection of demyelination in the brain from any previous myelin damage. It should be emphasized that a physiological myelin repair mechanism, i.e., remyelination of damaged myelin sheaths by resident perineural mature oligodendrocytes in proximity to the sheath can be activated following myelin damage, but rarely culminates in normal functional levels, particularly in aged patients in whom the oligodendrocytes function poorly.

The current FDA approved drugs for remyelinative therapy were initially targeted to slow the rate of relapse and/or the recurrence or accumulation of disability due to damaged sheath-induced axonal dysfunction and the scarring that occurs following demyelination. When damage to myelin is severe, axonal damage-induced disability can become permanent. The strategy of current and past treatments of anticipated, incipient or progressive demyelinating disease is to reduce, or stop a myelin-toxic immune response. Novel current experimental procedures include stem cell transplants, drugs targeting myelin-repair biochemical pathways, and drugs inducing oligodendrocyte progenitor cells. Planned preventative, and therapeutic measures to address radiation-induced demyelination require controlled clinical trials to determine their efficacy. Whether remyelination measures affect growth of residual tumors following incomplete therapy, is unknown. Ruling out this concern will require in vitro or in vivo studies in heterotransplanted tumor models in immunocomprimized mice models prior to clinical trials.

Among remyelinating measures are correction of mineral and vitamin deficiency states based upon extrapolating data from infants and children who develop demyelinating diseases. If they were deficient in minerals like copper at an early stage in development (13), or in B and D vitamins (14), 
supplementation with copper, and B and D vitamins in patients undergoing cerebral radiation may have a beneficial effect.

Patients nutritional status can also have a supportive effect upon the metabolic function of myelin via mitochondrial oxidative phosphorylation (Oxphos), which is the final biochemical pathway in the production of adenosine triphosphate. The myelin sheath layers and the axoplasm are connected by connexins suggesting a possible way to transfer adenosine triphosphate from the sheath to axon $(15,16)$. The energy supplied by adenosine triphosphate from the myelin sheath has a critical role in axon maintenance and function. In addition to nutritional deficiencies, alterations in myelin sheath metabolism by a variety of external chemical toxins and ionizing radiation form the etiopathogenesis of demyelinating and neurodegenerative disorders characterized by myelin sheath and axonal hypometabolism. Repair requires maintenance or restoration of the extramitochondrial oxphos functionality in myelin (17). Brain energy demand is a function of glucose and lactate catabolism which are the major cerebral energy substrates. Lactate, is transported exclusively by monocarboxylate transporters, and changes to these transporters alter lactate production. The most abundant lactate transporter in the central nervous system, monocarboxylate transporter 1 (MCT1) is highly enriched within oligodendroglia, and disruption of this transporter produces axon damage in animal and cell culture models. In addition, this same transporter is reduced in patients with amyotrophic lateral sclerosis, suggesting a role for oligodendroglial MCT1 in its pathogenesis (18). The role of MCT1 and amelioration, or prevention of radiation induced demyelination requires exploration. Because of the association of immune-based demyelination and surgery, chemotherapy and radiation, immune targeted treatment with lymphocyte CD-20 inhibitors such as Rituximab, or immune modulators, Interferon beta-1a, would be appropriate candidates to test. There is evidence that Glatiramer acetate converts the body's immune response from a Th1 type to a Th2 one and promotes suppressor T cells $(19,20)$. However, the use of this drug has led to rare cases of progressive multifocal leucoencephalopathy. Fingalimod is a compound that has shown efficacy in advanced clinical trials for the treatment of multiple sclerosis with therapeutic efficacy substantiated in phase 2 and 3 trials involving patients with relapsing or relapsing-remitting multiple sclerosis. Fingolimod phosphate initially activates lymphocyte S1P1 via high-affinity receptor binding, but subsequently induces S1P1 down-regulation that prevents lymphocyte egress from lymphoid tissues, thereby reducing auto-aggressive lymphocyte infiltration into the central nervous system. By activating sensors in lymph nodes which retain the white blood cells in the lymph nodes and lowering the number of auto-reactive $\mathrm{T}$ and $\mathrm{B}$ cells circulating in the blood, it prevents neuro-inflammation. The induced effects on remyelination and astrogliosis are mediated primarily through S1P3 and S1P5 indicating that it enhances nerve regeneration and remyelination. While the mechanism of action may differ, glatiramer and fingalimod may in rare cases also lead to progressive multifocal leukoencephalopathy. Further, the combination of fingolimod and radiotherapy has not been explored (21). Another measure to potentially reduce radiation-induced demyelination is recombinant human growth hormone, a pleiotropic hormone that functions in brain development, regulation of neuronal differentiation, modulation of brain neurotransmitters, and also in neural regeneration in animal models. Growth hormone administration following neural injury accelerates the recovery of neural functions. By inducing the maturation factor IGF-1, the function of remyelinating oligodendrocytes in multiple sclerosis patients is improved. In a clinical trial, 15 patients completed six months of rhGH treatment and exhibited no increase in clinical disease activity (22).

The immune response to tumor in the brain, and anti-tumor therapeutic measures reflects the complexity of the immune system. There is a subset of lymphocytes called myelinreactive Foxp ${ }^{+} \mathrm{CD}^{+} \mathrm{T}$ regs which may potentially enhance remyelination and ameliorate autoimmune disease (23).

However, there is also evidence that a different subset of Tregs may have negative effects on myelination and induce functional impairments (24). This is supported by evidence that transfer of expanded polyclonal Tregs does not affect ongoing autoimmune disease. Thus, the activity of distinct pools of transferred Treg cells as an effective therapeutic agent for remyelination depends upon their characteristics and relevance to the central nervous system for Tregs (25).

\section{Conclusion}

There is a wide spectrum of agents inducing demyelination in the central nervous system. Among these agents, tumoricidal ionizing radiation therapy intended for clinical benefit can simultaneously have severe damaging effects upon myelinated nerves. Un-mitigated radiation-induced damages of the myelin sheath, may result in a large number of chronic neurological disorders because of the intimate anatomic relationship and dependence of underlying axons on myelin energetic and trophic processes for efficient neural impulse conduction. Remediation of myelin damage and consequent recovery of normal neural function caused by ionizing radiation requires preventative or concomitant application of remyelinating strategies. To achieve this, there is a significant number of potentially effective drugs available to either prevent radiation-induced demyelination, limit ongoing demyelination, or stimulate remyelination. These drugs may have adverse effects and require crafting careful clinical strategies to determine which are 
significantly effective, and how they should be administered with reference to therapeutic radiation of the central nervous system so as to prevent exacerbation of the adverse effects or the development of additional neurological disorders.

\section{Conflicts of Interest}

The Author has no competing financial or commercial interests to declare.

\section{References}

1 Druckmann A: Schlafsucht als Folge der Roentegenbestrahlung: Beitrag zur strahlenem Pfindlichkeit des Gehirns. Strahlentherapie 33: 382-384, 1929.

2 Lampert P, Tom MI and Rider WD: Disseminated demyelination of the brain following Co60 (gamma) radiation. Arch Pathol 68: 322-330, 1959.

3 Rider WDJ: Radiation damage to the brain - a new syndrome. J Can Assoc Radiol 14: 67-69, 1963.

4 Nam JY and DeGroot JF: Treatment of glioblastoma. J Oncol Pract 13(10): 629-638, 2017.

5 DeGroot JF, Penas-Prado M, Mandel JJ, O’Brien BJ, Weathers SPS, Zhou S, Hunter K, Alfaro-Munoz K, Fuller GN, Huse J, Rao G, Weinberg JS, Prabhu SS, Ferguson SD, Yuan Y, Vence LM, Allison JP, Sharma P and Heimberger AB: Window-ofopportunity clinical trial of a PD-1 inhibitor in patients with recurrent glioblastoma. J Clin Oncol 36(15), 2018.

6 Nilsson J, Holgersson G, Carlsson T, Henriksson R, Bergström $\mathrm{S}$ and Bergqvist $\mathrm{M}$ : Incidence trends in high-grade primary brain tumors in males and females. Oncol Lett 13(4): 2831-2837, 2017.

7 Zhu H, Leiss L, Yang N, Rygh CB, Mitra SS, Cheshier SH, Weissman IL, Huang B, Miletic H, Bjerkvig R, Enger PØ, Li X and Wang J: Surgical debulking promotes recruitment of macrophages and triggers glioblastoma phagocytosis in combination with CD47 blocking immunotherapy. Oncotarget 8(7): 12145-12157, 2017.

8 Love S and Coakham HB: Trigeminal neuralgia: pathology and pathogenesis. Brain 124(Pt 12): 2347-2360, 2001.

9 Lampert PW and Davis RL: Delayed effects of radiation on the human central nervous system: 'early' and 'late' delayed reactions. Neurology 14: 912-917, 1964.

10 Lee Y, Morrison BM, Li Y, Lengacher S, Farah MH, Hoffman PN, Liu Y, Tsingalia A, Jin L, Zhang PW, Pellerin L, Magistretti PJ and Rothstein JD: Oligodendroglia metabolically support axons and contribute to neurodegeneration. Nature 487(7408): 443-448, 2012.

11 Ravera S, Bartolucci M, Adriano E, Garbati P, Ferrando S, Ramoino P, Calzia D, Morelli A, Balestrino M and Panfoli I: Support of nerve conduction by respiring myelin sheath: role of connexons. Mol Neurobiol 53(4): 2468-2479, 2015.
12 Vrdoljak E, Bill CA, Stephens LC, van der Kogel AJ, Ang KK and Tofilon PJ: Radiation-induced apoptosis of oligodendrocytes in vitro. Int J Radiat Biol 62(4): 475-480, 1992.

11 Asai A, Matsutani M, Matsuda T, Tanaka Y and Funada N: Radiation induced demyelination in Glioma therapy [Radiationinduced brain atrophy]. Brain Nerve 60(2): 123-129, 2008.

12 Asai A, Matsutani M, Kohno T, Nakamura O, Tanaka H, Fujimaki T, Funada N, Matsuda T, Nagata K and Takakura K: Subacute brain atrophy after radiation therapy for malignant brain tumor. Cancer 63(10): 1962-1974, 1989.

13 Klevay LM: Myelin and traumatic brain injury the copper deficiency hypothesis. Med Hypotheses 81(6): 995-998, 2013.

14 Black MM: Effects of vitamin B12 and folate deficiency on brain development in children. Food Nutr Bull 29: S126-S131, 2008

15 Ravera $S$ and Panfoli I: Role of myelin sheath energy metabolism in neurodegenerative diseases Neural Regen Res 10(10): 1570-1571, 2015.

16 Ravera S, Bartolucci M, Adriano E, Garbati P, Ferrando S, Ramoino P, Calzia D, Morelli A, Balestrino M and Panfoli I: Support of nerve conduction by respiring myelin sheath: role of connexons. Mol Neurobiol 53(4): 2468-2479, 2015.

17 Shapira $\mathrm{H}$ and Cock HR: Mitochondrial myopathies and encephalomyopathies. Eur J Clin Invest 29(10): 886-898, 1999.

18 Martinez BA: Lactate starved neurons in ALS. Dis Model Mech 5(6): 711-712, 2012.

19 Weber MS, Hohlfeld R and Zamvil SS: Mechanism of action of glatiramer acetate in treatment of multiple sclerosis. Neurotherapeutics 4(4): 647-653, 2007.

20 Milo R, Panitch H, Swoveland P and Bever CT Jr.: Glatiramer acetate blocks the activation of THP-1 cells by interferongamma. Eur J Pharmacol 342(2-3): 303-310, 1998.

21 Miron VE, Ludwin SK, Darlington PJ, Jarjour AA, Soliven B, Kennedy TE and Antel JP: Fingolimod (FTY720) enhances remyelination following demyelination of organotypic cerebellar slices. Am J Pathol 176(6): 2682-2694, 2010.

22 Stoppe M, Ettrich B, Thomae E, Lobsien D, Kratzsch J, Hasenclever D and Bergh FT: Recombinant human growth hormone for remyelination in MS: Results of a pilot trial in patients with chronically delayed VEP (P5.344). Neurology 88 : P5.344, 2017.

23 O'Conner RA and Anderton SM: Multifaceted control of autoaggression: Foxp3+ regulatory T cells in murine models of organ specific autoimmune disease. Cell Immunol 251: 8-18, 2008.

24 Anderton SM and Liblau RS: Regulatory T cells in the control of inflammatory demyelinating diseases of the central nervous system. Curr Opin Neurol 21: 248-254, 2008.

25 Stephens LA, Malpas KH and Anderton SM: Curing CNS autoimmune disease with myelin-reactive Foxp3+Treg. Eur J Immunol 39(4): 1108-1117, 2009.

Received June 27, 2018

Revised July 11, 2018

Accepted July 12, 2018 